\title{
Segmentação automática 2D de vias aéreas em imagens de tomografia computadorizada do tórax
}

Tarique da Silveira Cavalcante*, Paulo César Cortez, Thomaz Maia de Almeida, John Hebert da Silva Felix, Marcelo Alcantra Holanda

Resumo Introdução: Grande parte da população mundial é afetada por doenças pulmonares, como é o caso das broncopatias constituídas pela asma, bronquiectasia e a bronquite. O diagnóstico de broncopatias é baseado no estado das vias aéreas. Neste sentido, a segmentação automática das vias aéreas em imagens de Tomografia Computadorizada (TC) do tórax é uma etapa fundamental para auxílio ao diagnóstico dessas doenças. Métodos: O presente trabalho avalia algoritmos e desenvolve métodos de segmentação automática das vias aéreas 2D. Tais métodos são compostos por algoritmos de detecção de vias aéreas, sendo estes rede neural Multilayer Perceptron (MLP) e Análise de Densidades Pulmonares (ADP), e por algoritmos de segmentação de vias aéreas, sendo estes Crescimento de Região (CR), Método de Contornos Ativos (MCA) Balão e Topológico Adaptativo. Resultados: Os resultados foram obtidos em três etapas: análise comparativa entre os algoritmos de detecção MLP e ADP, com um padrão-ouro adquirido por três médicos com expertise em imagens de TC do tórax; análise comparativa entre algoritmos de segmentação MCA balão, MCA topológico adaptativo, MLP e CR; e avaliação das possíveis combinações entre os algoritmos de detecção e segmentação, resultando no método completo para segmentação automática das vias aéreas em 2D. Conclusão: A baixa incidência de falso-negativo e a redução significativa de falso-positivo, resulta em coeficiente de similaridade e sensibilidade superior a $91 \%$ e $87 \%$ respectivamente, para uma combinação dos algoritmos, com qualidade de segmentação satisfatória.

Palavras-chave Segmentação de vias aéreas, Tomografia computadorizada, Método de contornos ativos, Análise de densidades pulmonares.

\section{Automatic $2 D$ segmentation of airways in thorax computed tomography images}

Abstract Introduction: Much of the world population is affected by pulmonary diseases, such as the bronchial asthma, bronchitis and bronchiectasis. The bronchial diagnosis is based on the airways state. In this sense, the automatic segmentation of the airways in Computed Tomography (CT) scans is a critical step in the aid to diagnosis of these diseases. Methods: This paper evaluates algorithms for airway automatic segmentation, using Neural Network Multilayer Perceptron (MLP) and Lung Densities Analysis (LDA) for detecting airways, along with Region Growing (RG), Active Contour Method (ACM) Balloon and Topology Adaptive to segment them. Results: We obtained results in three stages: comparative analysis of the detection algorithms MLP and LDA, with a gold standard acquired by three physicians with expertise in CT imaging of the chest; comparative analysis of segmentation algorithms ACM Balloon, ACM Topology Adaptive, MLP and RG; and evaluation of possible combinations between segmentation and detection algorithms, resulting in the complete method for automatic segmentation of the airways in 2D. Conclusion: The low incidence of false negative and the significant reduction of false positive, results in similarity coefficient and sensitivity exceeding $91 \%$ and $87 \%$ respectively, for a combination of algorithms with satisfactory segmentation quality.

Keywords Airways segmentation, CT, Active contours method, Lung densities analysis. 


\section{Introdução}

O estudo de doenças pulmonares, bem como de seus tratamentos, têm muita importância, pois dentre as diversas doenças que afetam a população mundial, grande parte são ocasionadas nos pulmões, como é o caso das broncopatias, causadas por afecções das vias aéreas, como a asma, a bronquiectasia, e a Doença Pulmonar Obstrutiva Crônica (DPOC) (Nohama et al., 2012).

A Organização Mundial de Saúde (OMS) estima que existam 300 milhões de pessoas em todo o mundo acometidas pela asma. Esta doença causará cerca de 250 mil mortes anualmente em todo o mundo. A OMS também estima que 210 milhões de pessoas sejam portadoras de DPOC em todo o mundo e que o número de mortes por DPOC aumentará 30\% nos próximos dez anos. Além disso, estima-se que em 2030 a DPOC será a $3^{\mathrm{a}}$ causa de morte no mundo (Campos e Lemos, 2009; Organização..., 2008).

Em imagens de Tomografia Computadorizada (TC), as vias aéreas se apresentam em diversos tamanhos $\mathrm{e}$ formatos, além de, em alguns casos, apresentarem com pouca clareza suas forma e limites devido ao efeito de volume parcial. Por estes motivos, a segmentação das vias aéreas não se revela uma atividade trivial.

Dentre as diversas técnicas de segmentação, presentes na literatura, aplicadas às vias aéreas, a mais comum é o crescimento de região (CR). Mori et al. (2000) segmentam as vias aéreas somente através de CR, técnica limitada por vazamento em caso de ocorrência de descontinuidades no contorno. Tais limitações do CR motivam pesquisadores a integrar novas técnicas ao $\mathrm{CR}$ sempre com o intuito de diminuí-las.

Trabalhos recentes propõem alterações no CR básico a fim de obter melhores resultados na segmentação das vias aéreas, como Fabijanska (2009), que utiliza $\mathrm{CR}$ em duas fases, e Mendoza et al. (2009) e Weinheimer et al. (2009), que utilizam CR adaptativo. Porém, todos continuam com os resultados limitados pelo vazamento no decorrer da segmentação. Tschirren et al. (2009) utilizam somente $\mathrm{CR}$ e desenvolvem ferramentas para correção manual de erros, tornando o usuário final responsável por corrigir os erros obtidos durante a segmentação.

Bartz et al. (2003), Born et al. (2009) e Busayarat e Zrimec (2005) propõem a segmentação das vias aéreas através de $\mathrm{CR}$ e comparação de modelos. Os resultados são adequados, desde que não exista mais de uma artéria próxima à via aérea (artérias adjacentes ambíguas), além de apresentarem baixa precisão em pequenos brônquios e artérias.
Rizi et al. (2008a) utilizam conectividade fuzzy para diminuir as falhas do algoritmo CR e otimizar a segmentação das vias aéreas adicionando informação de forma com o intuito de continuar reduzindo os vazamentos no algoritmo $\mathrm{CR}$, mas não o eliminando por completo (Rizi et al., 2008b).

Felix et al. (2007), através de morfologia matemática e CR, segmentam sem distinção todas as estruturas internas do pulmão. Kiraly et al. (2002), utilizando as mesmas técnicas com foco em vias aéreas, segmentam apenas parte da árvore aérea, assim como Irving et al. (2009) que além de CR e morfologia, também utilizam a técnica de limiarização. Lo e Bruijne (2008) e Lo et al. (2010) segmentam as vias aéreas também com a técnica de CR e com um classificador KNN (K-nearest neighbor). Este classificador auxilia na segmentação, verificando se a mesma está correta ou se houve vazamentos.

Todos os trabalhos citados utilizam CR e grande parte destes, sempre com o intuito de reduzir a influência das limitações do CR, empregam outras técnicas que funcionam apenas como mecanismos de detecção e/ou prevenção de vazamento, o que não o evita por completo.

Bauer et al. (2009), que segmentam as vias aéreas utilizando GVF, Gradient Vector Flow (Xu e Prince, 1998), apresentam resultados com $60 \%$ de acerto e alguns trechos representados por aproximações. Fato este que ocorre devido a minúscula dimensão da via aérea que não é preservada ao se aplicar o GVF. Cheng et al. (2007) e Shi et al. (2006) utilizam Métodos de Contornos Ativos (MCA), porém, para segmentar somente a via aérea superior (traqueia) em imagens de TC do complexo maxilo-facial.

Além disso, todos os trabalhos de segmentação da árvore brônquica anteriormente citados realizam segmentação 3D, e para isso, necessitam obrigatoriamente de exames completos, realizados em tomógrafos multidetectores, com espessura de corte mínima. Estimando que o comprimento médio de um pulmão seja de $30 \mathrm{~cm}$, estes exames contêm cerca de 300 imagens para espessura de corte de 1,0 $\mathrm{mm}$ e 600 imagens para espessura de corte de $0,5 \mathrm{~mm}$, podendo variar de acordo com o tamanho do pulmão do paciente (Irion et al., 2007; Nakano et al., 2000). A realização deste tipo de exame requer equipamento específico, possui custo maior e submete o paciente a um alto índice de radiação quando comparados a exames simples com poucas imagens geradas. Isto desmotiva ou impossibilita sua realização em alguns casos.

Uma importante contribuição deste trabalho revela-se na possibilidade de análise das vias aéreas em imagens singulares. Isto se dá pelo fato de que 
este trabalho realiza a segmentação automática das vias aéreas em 2D, ou seja, uma simples imagem (secção do pulmão) é suficiente para que as vias aéreas representadas nesta sejam analisadas. Logo não há necessidade de exames com inúmeras imagens representativas de toda a caixa torácica do paciente. Ressalta-se, que trabalhos sobre segmentação automática 2D das vias aéreas são raros na literatura.

Nesse sentido, com a finalidade de obter segmentações precisas de vias aéreas, possibilitando a extração de atributos para auxílio ao diagnóstico, este trabalho propõe métodos inéditos para segmentação automática de vias aéreas em imagens 2D de TC. Vale ressaltar que existem dois tipos de TC, a single-detector spiral e a multidetector spiral (Webb et al., 2006). Apenas as TCs multidetector spiral ou helicoidal multidetectores são capazes de realizar a aquisição volumétrica da caixa torácica completa, logo os métodos propostos neste trabalho podem ser utilizados tanto em seções da imagem 3D da TC multidetector spiral, como nas imagens 2D que são geradas na TC sigle-detector spiral.

\section{Métodos}

Este trabalho propõe uma metodologia para realizar segmentações de vias aéreas em imagens 2D de TC do tórax. Tal metodologia é composta por duas etapas, a primeira consiste na deteç̧ão, ou seja, na localização das vias aéreas na imagem, o resultado desta etapa consiste na indicação de onde inicializar os algoritmos de segmentação, que por sua vez compõem a segunda etapa, responsável por realizar a segmentação das vias aéreas localizadas.

Neste sentido, para realizar a inicialização dos algoritmos de segmentação, são avaliados dois algoritmos de detecção das vias aéreas, sendo estes a rede MLP e o algoritmo de Análise de Densidades Pulmonares (ADP) (Cavalcante et al., 2011).

Com o intuito de eliminar a principal limitação do algoritmo de CR para segmentação de vias aéreas são avaliados os algoritmos MCA Balão, MCA Topológico Adaptativo e rede neural perceptron multicamadas (MLP) como alternativas para segmentação das vias aéreas em imagens $2 \mathrm{D}$ de TC.

Um diagrama geral da metodologia proposta é ilustrado na Figura 1. Para realizar a segmentação automática das vias aéreas pulmonares, inicialmente é necessário adquirir imagens de TC do tórax. Em seguida, as vias aéreas são automaticamente detectadas e posteriormente segmentadas. Cada combinação entre algoritmos de detecção e segmentação dá origem a um método distinto de segmentação automática de vias aéreas.

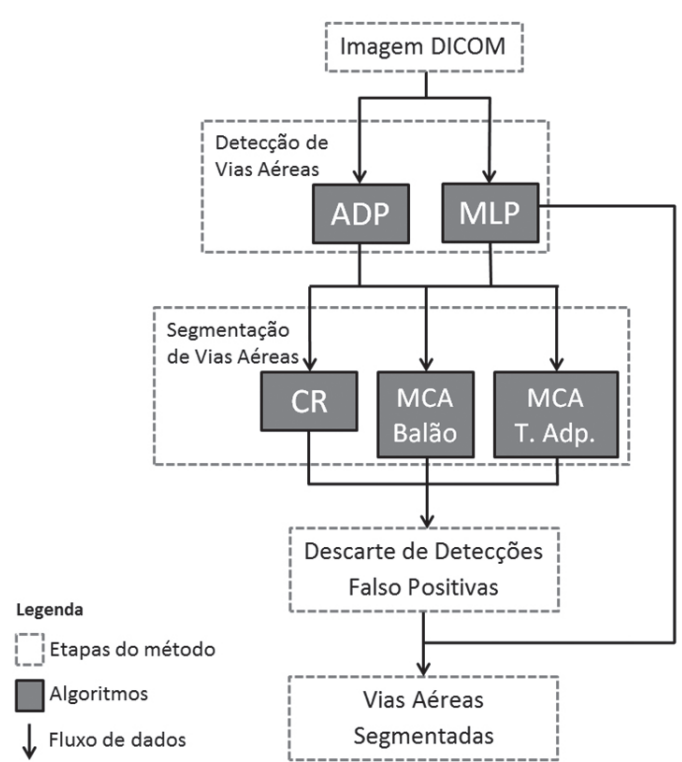

Figura 1. Diagrama geral dos métodos de segmentação automática das vias aéreas.

Figure 1. Diagram of general methods for automatic airway segmentation.

Nesta seção é descrita a etapa de aquisição de imagens de TC, os algoritmos, os parâmetros e as configurações propostas para deteç̧ão e segmentação das vias aéreas, bem como os procedimentos para avaliação e validação destes algoritmos.

\section{Aquisição de imagens}

O processo de aquisição de imagens utiliza dois tipos de tomografia computadorizada, TC multidetectores e Tomografia Computadorizada de Alta Resolução (TCAR). As imagens de TC multidetectores são adquiridas em um tomógrafo GE MEDICAL SYSTEMS modelo LightSpeed16, enquanto as imagens de TCAR são obtidas em um tomógrafo TOSHIBA modelo Auklet. O tamanho da imagem reconstruída é de $512 \times 512$ pixels. As imagens são quantificadas em 16 bits e armazenadas no padrão DICOM (Felix et al., 2009, 2010; Holanda et al., 2010).

As imagens utilizadas neste trabalho fazem parte de um banco de imagens construído a partir de estudos anteriores e aprovadas pelo Comitê de Ética em Pesquisa do hospital onde foram realizados os exames (Felix et al., 2009, 2010; Holanda et al., 2010). Tal banco de imagens é composto por 72 imagens de voluntários sadios e 20 imagens de paciente com DPOC. Em relação ao tipo de corte este banco de imagens contém 11 imagens no corte sagital, 12 imagens no corte coronal, 17 imagens no corte axial em nível de base, 24 imagens no corte axial em 
nível de hilo e 28 imagens no corte axial em nível de ápice. Tal diversidade de imagens exige dos métodos elevado grau de generalização, uma vez que estes devem apresentar resultados adequados independente do tipo de imagem.

\section{Avaliação dos métodos}

O processo de segmentação automática, para melhor avaliação, é dividido em duas etapas. A primeira etapa é a detecção automática das vias aéreas. O resultado da detecção é utilizado como parâmetro de entrada para a segunda etapa, que consiste na segmentação propriamente dita.

A validação de acurácia e precisão de um algoritmo computacional é uma etapa extremamente importante e nem sempre trivial, principalmente quando o algoritmo é aplicado na área médica. Neste sentido, o desafio desta etapa é a identificação correta de um padrão-ouro, ou seja, de um parâmetro de referência (Jannin et al., 2006; Zaidi e Naqa, 2010).

No caso da validação de algoritmos aplicados em imagens médicas, alguns autores chegam a um padrão-ouro por meio da expertise de médicos especialistas. Zaidi e Naqa (2010) e Zijdenbos et al. (1994) validam algoritmos com base na comparação visual entre os resultados apresentados e segmentações manuais realizadas por médicos especialistas. Sardanelli et al. (2004) avalia o algoritmo com base na atribuição de notas de dois radiologistas aos resultados obtidos.

Neste trabalho, para obtenção do padrão-ouro, um total de 132 imagens, representativas de todas as regiões dos pulmões de voluntários sadios e pacientes com DPOC são analisadas por três médicos especialistas. Sendo um pneumologista e dois radiologistas. Ressalta-se que os médicos envolvidos possuem vasta experiência acadêmica e clínica, além de expertise em análise de imagens de TC do tórax.

Sabe-se que as vias aéreas são classificadas por geração. Após a traqueia, cada ramificação das vias aéreas dá origem a uma nova geração, ou seja, as vias aéreas geradas a partir da ramificação da traqueia são vias aéreas da primeira geração. A ramificação das vias aéreas da primeira geração geram vias aéreas de segunda geração e assim por diante. Dessa forma, todas as vias aéreas até a quarta geração e a traqueia, cortadas axialmente são assinaladas individualmente por cada um dos médicos. Em seguida, é realizada uma comparação entre as imagens analisadas por cada médico, de tal forma, que aquelas que coincidem perfeitamente são classificadas como concordantes. Enquanto que as imagens que não apresentam as mesmas vias aéreas assinaladas são reapresentadas para os médicos que, em comum acordo, definem quais estruturas, assinaladas ou não, são vias aéreas.

\section{Detecção de vias aéreas}

Para detectar as vias aéreas são avaliados dois algoritmos. Um baseado em rede MLP e o outro em ADP. A avaliação de ambos os algoritmos dar-se pela comparação dos resultados com o padrão-ouro, obtidos por médicos especialistas.

Com base nas vias aéreas discriminadas no banco de imagens pelos médicos (padrão-ouro) é definida a quantidade total de vias aéreas, a quantidade de vias aéreas localizadas corretamente, a quantidade de vias aéreas não localizadas (falso-negativo) e de outras áreas identificadas como via aérea pelos algoritmos (falso-positivo).

\section{Segmentação de vias aéreas}

Na etapa de segmentação das vias aéreas são avaliados quatro algoritmos, CR, MLP, MCA balão e MCA topológico adaptativo. A avaliação adotada para esta etapa baseia-se na avaliação qualitativa de segmentação descrita por Gonzalez e Woods (2008). Deste modo, são adotadas as cinco categorias definidas por Gonzalez e Woods (2008) descritas a seguir:

- A - altíssima qualidade, tão bom quanto se poderia desejar;

- B - qualidade aceitável, com a presença de pequenos erros;

- C - média qualidade, com a presença de erros grosseiros;

- D - baixa qualidade, apenas uma pequena parcela do objeto de interesse é segmentada; e

- E - não segmenta parte nenhuma do objeto de interesse ou o objeto de interesse não é representativo na região segmentada.

Com o intuito de avaliar os algoritmos adequadamente, a classificação dos resultados é realizada, por meio de análise junto a um médico pneumologista.

\section{Análise de Densidades Pulmonares (ADP)}

Em imagens de TC do tórax, um pulmão com todas as suas estruturas é representado em unidades Hounsfield (UH) de -1000 a $+100 \mathrm{UH}$. Esta faixa pode ser subdividida de forma que identifique estruturas e regiões pulmonares. Deste modo, as principais faixas de densidade para caracterização de regiões pulmonares são: de -1000 a -900 UH para áreas hiperaeradas; de -900 a -500 UH para áreas normalmente aeradas; de -500 a -100 UH para áreas pouco aeradas; e de -100 a 100 UH para áreas não aeradas (Felix et al., 2009; Vieira et al., 1998). 
É possível identificar, por meio da análise dos valores de densidade em UH em uma determinada região quais estruturas pertencem às vias aéreas. Após avaliar, em imagens com resolução $512 \times 512$ pixels, o desempenho de diversas dimensões de máscaras para análise desta vizinhança, observa-se que a eficácia desta aumenta quando a dimensão da máscara é próxima das dimensões de vias aéreas (Cavalcante et al., 2011).

Para se determinar a localização da via aérea é necessário calcular os percentuais de cada uma das faixas de aeração anteriormente especificadas. Para isto, assume-se que existe um conjunto de classes $c_{i}$, em que $i=0,1,2$ e 3 , na ordem em que as faixas de aeração foram apresentadas. Neste sentido, considerando $D(x, y)$ uma matriz de densidades pulmonares, representando uma imagem de TC, tem-se a função $f_{i}(x, y)$ que determina a quantidade de pontos com densidades presente em cada classe $c_{i}$, dada por (Cavalcante et al., 2011; Rebouças Filho et al., 2011)

$$
f_{i}(x, y)=\sum_{l=-k}^{k} \sum_{m=-k}^{k} R(x-l, y-m)
$$

sendo $k$ o raio da região a ser analisada e $R$ dado por

$$
R(x, y)=\left\{\begin{array}{l}
1, l_{\text {inf }}\left(c_{i}\right)<D(x, y)<l_{\text {sup }}\left(c_{i}\right) \\
0, \text { caso contrário }
\end{array}\right.
$$

em que $l_{\text {inf }}\left(c_{i}\right)$ e $l_{\text {sup }}\left(c_{i}\right)$ representam os limites inferior e superior da faixa de densidade da classe $c_{i}$

A partir do valor de $f_{i}(x, y)$, dado pela Equação 1, calcula-se o percentual $P_{i}$ de cada classe $i$ dado por

$$
P_{i}=\frac{f_{i}(x, y)}{\sum_{i=0}^{3} f_{i}(x, y)}
$$

Deste modo, pode-se obter uma imagem binária $g(x, y)$ dada por (Cavalcante et al., 2011)

$$
g(x, y)=\left\{\begin{array}{l}
1, \text { para } P_{3}+P_{2}>0,1 \text { e } P_{0}>0,05 \\
0, \text { caso contrário }
\end{array}\right.
$$

A imagem obtida através da função $g(x, y)$ não apresenta uma segmentação completa das vias aéreas, entretanto faz sua rotulação.

\section{Rede neural perceptron multicamada}

Este tipo de rede possui duas fases distintas: a de treinamento e a de execução. A fase de treinamento é supervisionada, ou seja, durante o treinamento é apresentado na entrada da rede um conjunto de parâmetros de exemplos, cujas saídas são conhecidas. Em seguida, o erro obtido para cada exemplo é utilizado para atualização dos pesos (Haykin, 1998). Durante a fase de execução, conjuntos de parâmetros cujas classes não são conhecidas são apresentados na entrada da rede, com os pesos definidos pelo treinamento, e posteriormente classificados.

A rede MLP utilizada adota o gradiente descendente de retro propagação do erro na fase de treinamento.
Esta rede fornece uma classificação para cada um dos pixels da imagem de entrada, ou seja, a resposta final da rede determina se um pixel específico faz ou não parte de uma via aérea.

Para a fase de treinamento, são selecionados manualmente pixels que, de acordo com o padrão-ouro, correspondem a vias aéreas. Estes pixels recebem o rótulo de "via aérea". Em seguida, pixels das demais estruturas que, de acordo com o padrão-ouro, não correspondem a vias aéreas são selecionados manualmente e recebem rótulo de "demais estruturas".

Cada pixel selecionado consiste em uma amostra que possui seis descritores, sendo estes as duas coordenadas do pixel na imagem e os 4 percentuais de faixas de densidade de sua vizinhança calculados com base na Equação 3. Por fim, é composto um banco de dados formado pelos descritores dos pixels selecionados. Em média, para compor o banco de dados, 60 pixels por imagem são selecionados, representando as vias aéreas e outras estruturas presentes na imagem.

A dimensão da vizinhança considerada para cálculo do percentual das faixas de densidade deve ser proporcional ao ruído da imagem, ou seja, quanto maior o ruído, maior a dimensão da vizinhança. Uma vez que o ruído em UH não se enquadra em nenhuma faixa de densidade, sua ocorrência em uma dada região diminui a quantidade de informação útil, sendo então necessário aumentar a área considerada a fim de obter-se quantidade adequada de pixels que sejam classificados nas faixas de densidade, possibilitando o cálculo de percentuais representativos.

A rede MLP apresenta a camada de saída com um neurônio, pois possui somente um padrão-a ser definido, ou seja, uma saída para indicar se o elemento faz ou não parte de uma via aérea. A determinação da quantidade de neurônios que deve ser utilizado na camada oculta, é uma tarefa complexa (Murata et al., 1994), em que usualmente faz-se uso de heurísticas. Sabe-se que existe um número ótimo para esta quantidade de forma a minimizar erros durante sua fase de execução. Todavia, como esta determinação não é objetivo deste trabalho, sendo recomendada para trabalhos futuros, adotou-se, com base em testes preliminares realizados, a configuração para a rede com 6 entradas, 10 neurônios na camada oculta e um neurônio na camada de saída.

Desta forma, as 6 entradas consistem nos descritores dos pixels anteriormente definidos. O neurônio da camada de saída indica a classificação final do pixel com uma saída positiva quando este pertence a uma via aérea e com uma saída negativa quando o pixel não pertence a nenhuma via aérea. A taxa de erro calculada durante a fase de treinamento 
é baseada na diferença entre o rótulo atribuído ao pixel pela rede neural e o rótulo presente no banco de dados. Ou seja, se os rótulos são os mesmos, independentemente de qual seja, é contabilizado um acerto, caso contrário, um erro é contabilizado. Por fim, a taxa de erro consiste no percentual de erros contabilizados em relação ao total de amostras do banco de dados.

\section{Crescimento de Região (CR)}

Para a aplicação da técnica de CR é necessário estabelecer dois parâmetros: a semente e a definição para crescimento (Gonzalez e Woods, 2008). Neste trabalho, as sementes são baseadas nas respostas dos algoritmos de detecção, ou seja, são os pixels considerados como parte de vias aéreas pelos algoritmos ADP ou MLP, de tal forma que, para cada região detectada o pixel de coordenada média se torna a semente. Desta forma, cada via aérea deve ser uma região segmentada.

A definição de crescimento adotada $P\left(R_{i}\right)$ consiste na proximidade dos níveis de cinza em UH e pode ser definida por

$P\left(R_{i}\right)=\left\{\begin{array}{l}1, \text { se }(1-t) d_{s}<d_{p} \leq(1+t) d_{s} \\ 0, \text { caso contrário }\end{array}\right.$

em que $t$ é a tolerância aceitável para os níveis de densidade, assumindo valores entre 0 e $1, d_{s}$ é o valor da densidade da semente e $d_{p}$ o valor da densidade do pixel analisado, ambos em escala UH. A utilização de uma faixa de tolerância, como a definida na Equação 5, torna-se importante pelo fato de possibilitar a adesão de grupos de pixels com propriedades similares às da semente.

\section{Métodos de Contornos Ativos (MCA)}

O algoritmo do MCA proposto por Kass et al. (1988) consiste inicialmente em criar uma curva em torno do objeto de interesse. Esta curva é conduzida, através de forças, até as bordas do objeto e evolui então, de tal modo, que a sua energia diminui a cada nova iteração. O MCA consiste na parametrização $2 \mathrm{D}$ de uma curva geométrica da forma

$\left\{\begin{array}{l}{[0,1] \rightarrow \mathbb{R}^{2}} \\ s \rightarrow c(s)=[x(s), y(s)]\end{array}\right.$

A função energia do contorno ativo é descrita por (Kass et al., 1988)

$E_{s}=\int_{0}^{1}\left\{E_{\text {int }}[c(s)]+E_{\text {ext }}[c(s)]\right\} d s$

em que $c(s)$ é a curva, $E_{s}$ é a energia total do contorno, $E_{\text {ext }}$ a energia externa e $E_{\text {int }}$ a energia interna.

A energia externa $E_{e x t}$ representa a parcela da energia composta por forças externas, ou seja, forças que não dependem de qualquer característica do contorno, mas de propriedades da imagem. Neste sentido, operadores de gradiente são amplamente usados para determinar a energia externa dos MCAs (Nixon e Aguado, 2012). A energia interna $E_{\text {int }}$ diz respeito à fração da energia do MCA composta pelas forças internas. Estas dependem diretamente do formato do contorno e é definida por

$E_{\text {int }}\left(c_{i}\right)=\alpha F_{\text {cont }}\left(c_{i}\right)+\beta F_{\text {curv }}\left(c_{i}\right)$

em que $F_{\text {cont }}$ é a força de continuidade e consiste na primeira derivada do contorno, $F_{\text {curv }}$ é a força de curvatura que consiste na segunda derivada do contorno, e $\alpha$ e $\beta$ são parâmetros que ponderam as forças de continuidade e curvatura, respectivamente (Kass et al., 1988).

MCAs são aplicados com sucesso em vários problemas de visão computacional, tais como a detecção de bordas e rastreamento de objetos, entre outros. Ainda não é possível uma única solução para todas as aplicações, por causa da originalidade de cada problema caracterizado por imagens específicas em cada aplicação (Alexandria et al., 2011). O MCA tradicional, por exemplo, possui algumas limitações tais como as parametrizações da energia interna (ajuste dos valores $\alpha$ e $\beta$ ) que podem limitar sua flexibilidade, ou quando o contorno é inicializado no interior do objeto. Neste segundo caso, em regiões homogêneas o valor do gradiente é nulo, assim o contorno sofre influência apenas da energia interna, que tende a deixá-lo mais uniforme, e a minimizar sua área, resultando em seu colapso (Rebouças Filho et al., 2011).

Com o objetivo de eliminar limitações do MCA Tradicional, diversas pesquisas são realizadas propondo novos MCAs. Lee et al. (2007) e Rebouças Filho et al. (2011) aplicam o modelo level set, um dos MCAs geométricos, e o MCA Crisp, respectivamente, na segmentação dos pulmões em imagens de TC, enquanto Silva (2005) utiliza, para realizar a segmentação dos pulmões em imagens de TC, os contornos ativos paramétricos tradicionais e o Gradient Vector Flow (GVF).

\section{MCA balão}

A força balão foi proposta por Cohen (1991), com o intuito de solucionar a restrição do MCA tradicional, que entra em colapso quando é inicializado dentro do objeto. A força balão age expandindo ou comprimindo o contorno. Este fato revela-se em uma importante contribuição, pois quando a curva se encontra em regiões homogêneas da imagem, a força externa é nula e as únicas forças que devem interferir no contorno são as forças internas. Deste modo, a força balão deforma o contorno para que este se aproxime das 
bordas. No MCA balão, a energia total e a energia externa se mantêm iguais às do MCA tradicional. Entretanto, a energia interna é composta pela força de continuidade e pela força balão. Assim, para a energia interna tem-se (Cohen, 1991)

$$
E_{\text {int }}\left(c_{i}\right)=\alpha F_{\text {cont }}\left(c_{i}\right)+\beta F_{\text {bal }}\left(c_{i}\right)
$$

em que $F_{\text {cont }}\left(c_{i}\right)$ é a força de continuidade, $F_{b a l}\left(c_{i}\right)$ é a força balão; e $\alpha$ e $\beta$ são os parâmetros utilizados para ajustar estas forças.

\section{MCA topológico adaptativo}

O MCA topológico adaptativo é baseado no posicionamento de uma malha sobre a imagem, na qual o conjunto de pontos iniciais da curva se movimenta até encontrar as bordas do objeto desejado. Neste modelo, a energia externa se mantém igual ao modelo tradicional desenvolvido por Kass et al. (1988), e a energia interna é composta pela força de continuidade e pela força balão, assim como no MCA balão.

Os vértices do contorno do MCA topológico adaptativo são as interseções de um contorno inicial qualquer, sobre a malha triangular, com as arestas dos triângulos da malha. Os triângulos, cujas arestas possuem algum ponto do contorno, são denominados triângulos de borda (Mcinerney e Terzopoulos, 1999).

O MCA topológico adaptativo possui uma função característica definida por (Mcinerney e Terzopoulos, 1999)

$X: D \subset R^{2}[0,1]$

tal que

$$
\begin{aligned}
& X(v)=+1, \text { se } v \in 0 \\
& X(v)=-1, \text { se } v \notin 0
\end{aligned}
$$

em que $v$ é um vértice da malha triangular, $D$ é o domínio de interesse e $O$ é o contorno. Os vértices em que $X(v)=-1$, representam regiões não visitadas.

A função característica definida nas Equações 11 e 12 conduzem o contorno a uma condição de irreversibilidade, diminuindo sua oscilação. Assim, uma vez que o vértice recebe o valor positivo, é dito como visitado e permanece em tal estado, impossibilitando que o vértice faça parte do contorno novamente (Mcinerney e Terzopoulos, 1999).

\section{MCA para segmentação de vias aéreas}

A segmentação pelos MCAs balão e topológico adaptativo inicia-se a partir de uma curva inicial para cada via aérea. Esta curva é formada por quatro vértices (nós), que são definidos a um pixel de distância de um dado centroide. Este centroide é o próprio pixel, se este for único, ou uma coordenada média dos pixels obtidos da saída da etapa de detecção para cada via aérea, ou seja, cada curva segmenta apenas uma via aérea.

Os parâmetros a serem definidos para ambos os algoritmos são os pesos de cada força $\alpha$ e $\beta$, para as forças de continuidade, balão e energia externa respectivamente. De acordo com as análises realizadas em diversos trabalhos presentes na literatura (Dagher e Tom, 2008; Xu e Prince, 1998) e após uma série de testes, os valores dos termos de ponderação são iguais a 0,01 e 0,1 para $\alpha$ e $\beta$, respectivamente.

Para o MCA topológico adaptativo, além dos pesos e tamanho da máscara, é necessário definir a espessura da malha a ser utilizada. Como os nós da curva se posicionam apenas sobre as arestas das malhas, é necessário um espaçamento que proporcione definição suficiente, ou seja, que os volumes de controle sejam menores do que as vias aéreas.

Em imagens com a resolução padrão de $512 \times 512$ pixels, vias aéreas de até quarta geração possuem diâmetro maior do que 4 pixels. Assim, é adotada uma malha tradicional do tipo Coxeter-Freudenthal, com distância fixa entre vértices de 4 pixels, resultando em uma malha com 128 divisões em cada eixo, gerando um total de 32.768 volumes uniformes ao longo de toda a imagem.

\section{Resultados}

Para avaliar a eficácia dos métodos propostos, os resultados obtidos são divididos em três etapas: análise comparativa entre os algoritmos de detecção MLP e ADP, análise comparativa entre os algoritmos de segmentação MCA balão, MCA topológico adaptativo, MLP e CR, e avaliação das possíveis combinações entre os algoritmos de detecção e segmentação, resultando em métodos completos para segmentação automática das vias aéreas.

\section{Detecção de vias aéreas}

$\mathrm{Na}$ análise dos algoritmos de deteç̧ão é computado o coeficiente de similaridade e sensibilidade destes, visto que existem três tipos possíveis de resultados: Verdadeiro (V), Falso-Positivo (FP) e Falso-Negativo (FN). O caso verdadeiro (V) é para as estruturas que contém pixels detectados como vias aéreas pelo algoritmo e são classificadas como vias aéreas pelos médicos especialistas; FP para estruturas com conjunto de pixels detectados como vias aéreas pelo algoritmo, mas não consideradas vias aéreas pelos médicos especialistas; e as estruturas que não possuem nenhum pixel detectado como via aérea 
pelo algoritmo, mas são consideradas vias aéreas pelos médicos especialistas, correspondem aos FNs.

A partir da contabilização destes resultados, é possível definir o coeficiente de similaridade CS e sensibilidade $\mathrm{S}$, em termos de porcentagem dada por (Kaftan et al., 2008; Madeiro et al., 2009; Zou et al., 2004)

$$
\begin{aligned}
& C S=\frac{2 \times \mathrm{V}}{2 \times V+F N+F P} \times 100 \\
& S=\frac{\mathrm{V}}{V+F N} \times 100
\end{aligned}
$$

No conjunto de 132 imagens destinadas à fase de teste, 794 vias aéreas foram assinaladas pelos médicos especialistas.

Para o treinamento da rede MLP, seleciona-se aleatoriamente um conjunto representativo de 40 imagens, contendo imagens nos cortes coronal, sagital e axial, nas posições de ápice, hilo e base de voluntários sadios e pacientes com DPOC.

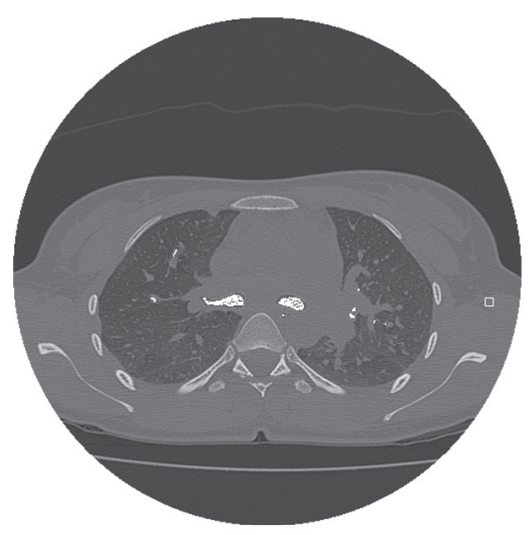

(a)

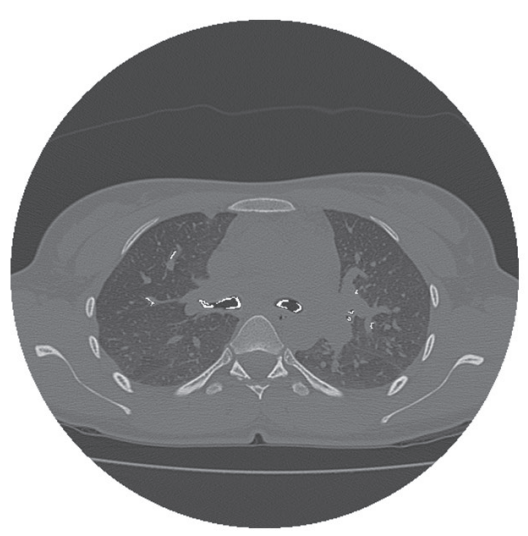

(c)
Após a fase de treinamento as 92 imagens restantes foram submetidas à rede MLP, que classifica cada pixel como pertencente ou não a uma via aérea. $\mathrm{O}$ mesmo conjunto de 92 imagens é também submetido ao algoritmo ADP, para obter resultados de detecção de vias aéreas. Na Figura 2 são ilustradas imagens de TC do tórax cujos pixels em branco são os pixels classificados como partes de vias aéreas.

Após obter a classificação das imagens, por meio de comparação com o padrão-ouro, constata-se para o algoritmo de detecção MLP a ocorrência de 444 detecções corretas, 62 detecções caracterizadas como FN e 145 detecções caracterizadas como FP, resultando, de acordo com as Equações 13 e 14 em um coeficiente de similaridade de $81,20 \%$, e sensibilidade de $87,74 \%$.

Com o algoritmo de detecção ADP os resultados obtidos são de 398 detecções corretas, 108 detecções caracterizadas como FN e 83 caracterizadas como FP, cujo coeficiente de sensibilidade corresponde a $80,64 \%$ e sensibilidade de $78,65 \%$.

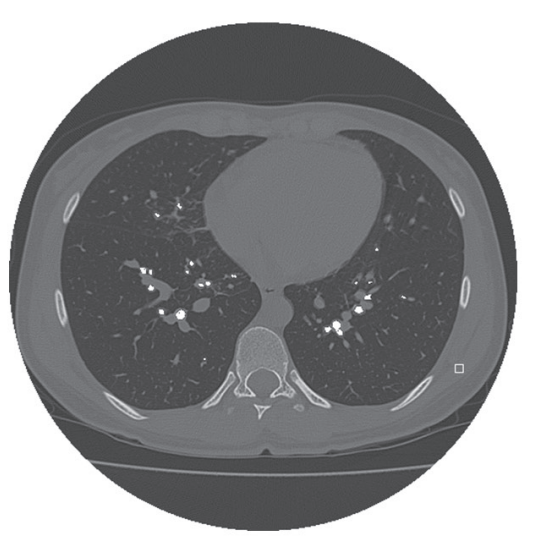

(b)

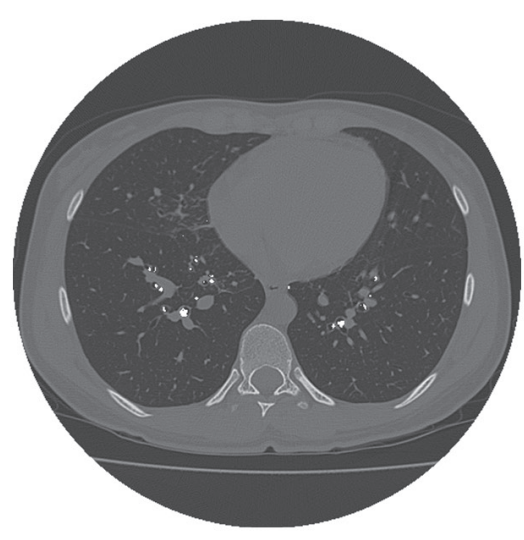

(d)

Figura 2. Imagens de TC com as vias aéreas detectadas assinaladas em branco: (a) e (b) detecção por MLP, (c) e (d) detecção por ADP. Figure 2. CT images with the detected airways marked in white: (a) and (b) detection by MLP, (c) and (d) detection by LDA. 
Para uma análise mais detalhada do conjunto de imagens testes são consideradas duas abordagens. Uma primeira abordagem que separa as imagens de voluntários sadios e de pacientes com DPOC; e a outra que divide as imagens de acordo com o tipo de corte, sendo estes coronal, sagital, axial em nível de ápice, axial em nível de hilo e axial em nível de base.

Na Tabela 1 são mostrados os resultados dos algoritmos MLP e ADP para as duas abordagens respectivamente. Os valores presentes nesta tabela consistem no número de ocorrências para cada tipo de detecção, coeficiente de similaridade e sensibilidade.

\section{Segmentação de vias aéreas}

A avaliação dos algoritmos de segmentação adotada neste trabalho baseia-se na avaliação qualitativa de segmentação descrita anteriormente. A inicialização dos MCAs e do CR é feita com base no resultado da detecção. A coordenada média de cada região, classificada pelos algoritmos de detecção como via aérea, é calculada. Os pixels destas coordenadas são usados como as sementes para o algoritmo de CR e a vizinhança destes são os contornos iniciais para os MCAs. A segmentação realizada por MLP consiste no resultado da etapa de deteç̧ão.

Exemplos de segmentação de vias aéreas por cada algoritmo avaliado são ilustrados na Figura 3. $\mathrm{O}$ resultado da avaliação realizada pelo médico especialista é mostrado na Tabela 2.

\section{Avaliação do método de segmentação automática de vias aéreas}

Nesta seção é avaliado o desempenho dos métodos de segmentação automática de vias aéreas, que consiste no conjunto de algoritmos de detecção e segmentação anteriormente avaliados. Por apresentar o mesmo comportamento do MCA balão e qualidade pior de segmentação, o MCA topológico adaptativo não foi considerado nesta etapa. Desta forma, são comparados os resultados das possíveis combinações, sendo estas: ADP e CR; ADP e MCA balão; MLP e CR; MLP e MCA balão; e somente MLP.

Ao empregar em conjunto os algoritmos de detecção e segmentação, inclui-se uma nova etapa responsável por descarte de detecções FP. A qualidade da segmentação avaliada na seção anterior, para cada um dos algoritmos, não se altera, porém o coeficiente de similaridade e sensibilidade dos algoritmos de detecção, calculada com base nas Equações 13 e 14, sofrem alterações.

Tais alterações ocorrem devido ao fato de ser possível detectar FP após a etapa de segmentação. Quando o MCA ou CR são inicializados em um FP, é apresentada uma segmentação com quantidade de pixels maior do que a quantidade de pixels presente nas vias aéreas.

Analisando o banco de 132 imagens de TC do tórax, constata-se que as maiores vias aéreas apresentam em média 1600 pixels. Neste sentido, adotando uma margem de segurança de $30 \%$, conclui-se que as segmentações que possuem quantidade de pixels maior do que 2080 podem ser automaticamente descartadas, o que reduz significativamente a quantidade de FP, aumentando o coeficiente de similaridade dos métodos. Resultados de segmentação do CR e do MCA balão quando estes são inicializados em detecções FP são ilustrados na Figura 4.

Devido a esta correção, algumas vias aéreas segmentadas pelo algoritmo $\mathrm{CR}$, categorizadas como D e E, são descartadas, pois as mesmas chegam a

Tabela 1. Resultados da detecção de vias aéreas para voluntários sadios, pacientes com DPOC e por tipos de corte.

Table 1. Results of airway detection for healthy volunteers, patients with COPD and by types of cut.

\begin{tabular}{|c|c|c|c|c|c|c|}
\hline Algoritmos de detecção & Categorias de análise & $\mathbf{V}$ & $\mathbf{F N}$ & $\mathbf{F P}$ & $\mathrm{CS}(\%)$ & $\mathrm{S}(\%)$ \\
\hline \multirow{7}{*}{ MLP } & Voluntários sadios & 371 & 57 & 98 & 82,7 & 86,7 \\
\hline & Pacientes com DPOC & 73 & 5 & 45 & 74,4 & 93,6 \\
\hline & Coronal & 41 & 6 & 15 & 79,6 & 87,2 \\
\hline & Sagital & 46 & 8 & 11 & 82,8 & 85,2 \\
\hline & Axial (ápice) & 103 & 21 & 45 & 75,7 & 83,1 \\
\hline & Axial (hilo) & 132 & 10 & 43 & 83,2 & 93,0 \\
\hline & Axial (base) & 122 & 17 & 31 & 83,5 & 87,8 \\
\hline \multirow{7}{*}{ ADP } & Voluntários sadios & 337 & 91 & 58 & 81,9 & 78,7 \\
\hline & Pacientes com DPOC & 61 & 17 & 25 & 74,4 & 78,2 \\
\hline & Coronal & 27 & 20 & 6 & 67,5 & 57,4 \\
\hline & Sagital & 33 & 21 & 8 & 69,5 & 61,1 \\
\hline & Axial (ápice) & 98 & 26 & 27 & 78,7 & 79,0 \\
\hline & Axial (hilo) & 123 & 19 & 25 & 84,8 & 86,6 \\
\hline & Axial (base) & 115 & 24 & 17 & 84,9 & 82,7 \\
\hline
\end{tabular}



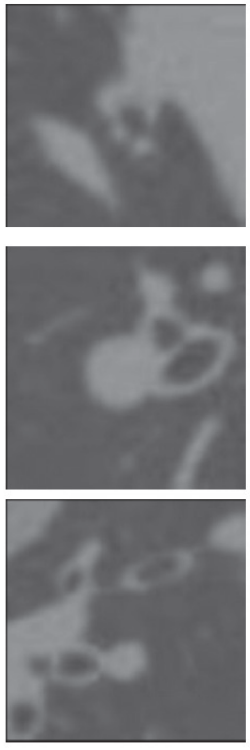

(a)
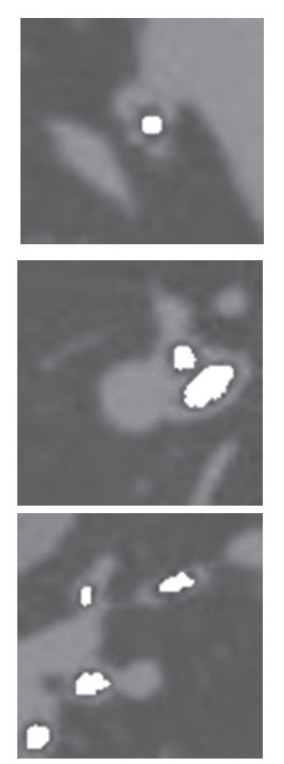

(b)
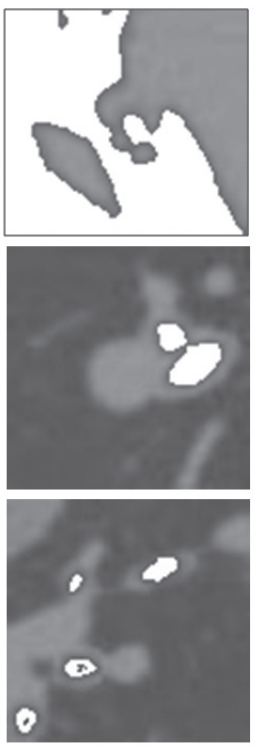

(c)
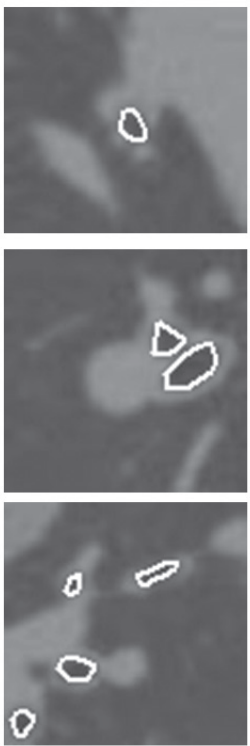

(d)
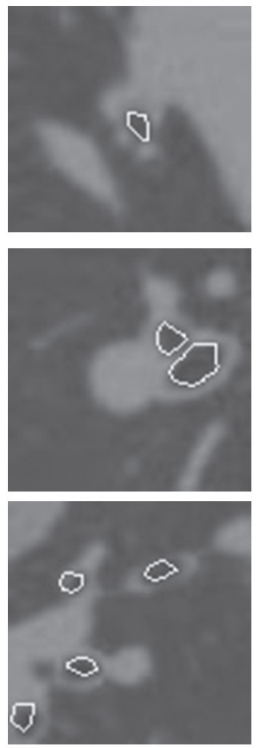

(e)

Figura 3. Resultados da segmentação das vias aéreas, nas colunas (a) recortes das imagens de TC; (b) segmentação por MLP; (c) segmentação por CR; (d) segmentação por MCA balão; e (e) segmentação por MCA topológico adaptativo.

Figure 3. Segmentation results of the airways, in columns (a) cutouts of CT images, (b) MLP segmentation, (c) RG segmentation; (d) ACM segmentation, and (e) ACM Topology Adaptive segmentation.

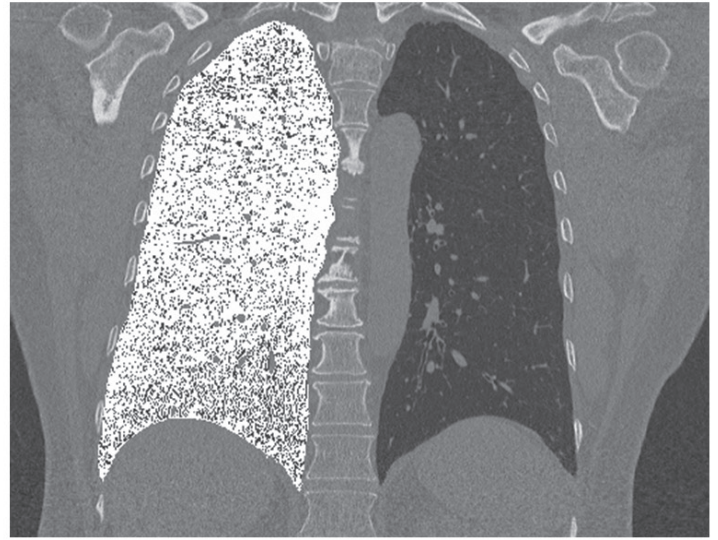

(a)

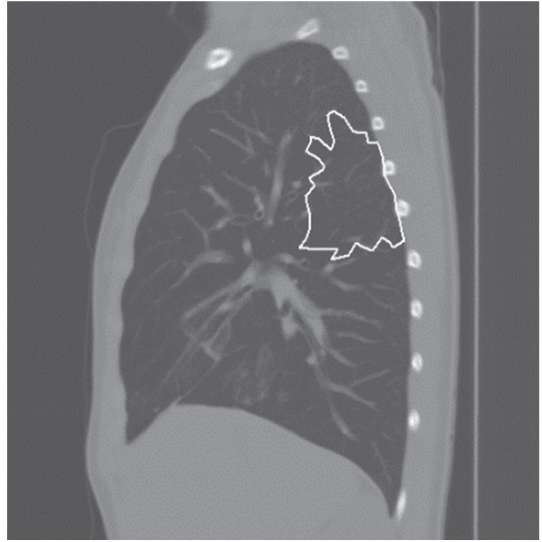

(b)

Figura 4. Resultado de segmentação inicializada com detecções FP, (a) CR; e (b) MCA balão.

Figure 4. Result of segmentation initialized with FP detections, (a) RG and (b) ACM balloon.

Tabela 2. Percentual da classificação da segmentação das vias aéreas. Table 2. Percentage classification of airway segmentation.

\begin{tabular}{lccccc}
\hline $\begin{array}{c}\text { Algoritmos de } \\
\text { segmentação }\end{array}$ & A & B & C & D & E \\
\hline MLP & 39,3 & 25,0 & 22,6 & 10,7 & 2,4 \\
CR & 35,7 & 35,7 & 11,9 & 1,2 & 15,5 \\
MCA balão & 30,9 & 52,4 & 15,5 & 1,2 & 0,0 \\
MCA topológico & 19,0 & 40,5 & 25,0 & 15,5 & 0,0 \\
\hline
\end{tabular}

apresentar quantidade de pixels excedente ao parâmetro adotado. Um exemplo desta ocorrência pode ser visualizado na coluna (c) da Figura 2.

$\mathrm{Na}$ Tabela 3 é contabilizada a quantidade de vias aéreas, a quantidade de detecções V, FN, FP, CS e S de cada combinação. Na Figura 5 são ilustrados gráficos comparativos entre os coeficientes de similaridade e taxa de sensibilidade, respectivamente, dos algoritmos 
Tabela 3. Resultados da segmentação automática de vias aéreas. Table 3. Results of the airways automatic segmentation.

\begin{tabular}{lccccc}
\hline \multicolumn{1}{c}{ Métodos } & V & FN & FP & CS (\%) & S (\%) \\
\hline ADP/CR & 321 & 185 & 7 & 76,9 & 63,4 \\
ADP/MCA & 398 & 108 & 18 & 86,3 & 78,6 \\
MLP/CR & 353 & 153 & 11 & 81,1 & 69,7 \\
MLP/MCA & 444 & 62 & 23 & 91,3 & 87,7 \\
MLP & 444 & 62 & 145 & 81,1 & 87,7 \\
\hline
\end{tabular}

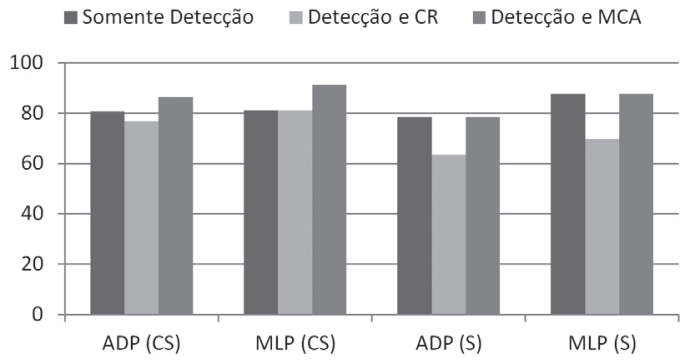

Figura 5. Gráfico com CS e S dos algoritmos de detecção ADP e MLP, e dos mesmos após integração com os algoritmos CR e MCA. Figure 5. Graphic showing the similarity coefficient and sensitivity of the LDA and MLP detection algorithms, and after integration of these algorithms with $R G$ and $A C M$.

de detecção e destes algoritmos integrados aos algoritmos de segmentação MCA, CR.

\section{Discussão}

Assim como na seção de resultados, as discussões dos resultados são divididas em três subseções: Detecção de Vias Aéreas, Segmentação de Vias Aéreas e Avaliação do Método de Segmentação Automática de Vias Aéreas. Este último consiste no método completo composto pelos algoritmos das duas subseções anteriores.

\section{Deteçã̃o de vias aéreas}

Com base nos resultados da MLP na Tabela 1, constata-se uma diferença de aproximadamente $7 \%$ entre as taxas de sensibilidade de voluntários sadios e pacientes com DPOC, porém o CS nas imagens de voluntários sadios é consideravelmente maior do que nas imagens de pacientes com DPOC. Pode-se atribuir parte dessa diferença à quantidade de detecções FP igual a $36,6 \%$ presente nas imagens de pacientes com DPOC, enquanto nas imagens de voluntários sadios as detecções FP representam apenas 18,6\%. Esse desempenho é plenamente justificável pelos níveis de densidade em UH, que em imagens de paciente com DPOC, tendem a ser menores, pois, o tecido pulmonar neste tipo de doença torna-se hiperaerado, apresentando valores em UH semelhantes às vias aéreas.
Ressalta-se que a ocorrência de FN nas imagens de pacientes com DPOC representa apenas $4 \%$ do total de detecções contabilizadas, enquanto em imagens de voluntários sadios as detecções FN somam 10\%.

Analisando a Tabela 2, percebe-se um desempenho uniforme nas imagens de corte sagital, axial em nível de base e axial em nível de hilo. Os coeficientes de similaridade das imagens em corte coronal e axial em nível de ápice apresentam valores menores devido à pequena dimensão e à pouca definição das vias aéreas neste nível.

Entretanto, a sensibilidade de $93 \%$ em imagens de corte axial em nível de hilo merece destaque, pois estas imagens contém uma quantidade elevada de vias aéreas. Este fato pode ser constatado pela soma dos valores de $\mathrm{V}$ e de FN na Tabela 2, Isto resulta num total de 142 vias aéreas assinaladas pelos médicos especialistas para esse tipo de imagem, neste conjunto.

O coeficiente de similaridade dos resultados do algoritmo ADP, na detecção de vias aéreas, está ligeiramente abaixo dos resultados obtidos com a rede MLP. Apesar disso, comparando os dados de ambos os algoritmos, percebe-se que o algoritmo ADP apresenta um número menor de detecções, em geral. A quantidade reduzida de detecções FP melhora o desempenho deste algoritmo, mas não o suficiente para contrapor o decréscimo nas detecções do tipo $\mathrm{V}$ apresentado em todos os casos.

Logo, os coeficientes de similaridades menores e a alta representatividade das detecções FN dos resultados do algoritmo ADP, são fatos suficientes para concluir que a detecção de vias aéreas baseada em MLP apresenta melhores resultados.

\section{Segmentação de vias aéreas}

Com base na Tabela 3, observa-se que a rede MLP e o CR são os dois algoritmos que apresentam os maiores percentuais de segmentação da categoria A, com $39,29 \%$ e $35,71 \%$, respectivamente. Por outro lado, os MCAs balão e topológico adaptativo que obtêm respectivamente $30,95 \%$ e $19,05 \%$ de segmentações da categoria $\mathrm{A}$, não realizam nenhuma segmentação da categoria E. Entretanto, a rede MLP obteve 2,38\% e o CR $15,48 \%$ de segmentações desta categoria.

Além disso, nota-se uma diferença relevante entre o desempenho dos MCAs balão e topológico adaptativo, uma vez que ambos são versões de um mesmo algoritmo. Apesar do MCA topológico adaptativo possuir algumas vantagens como a função característica e a adaptação à topologia, em relação ao MCA balão, seu desempenho está abaixo deste devido às pequenas dimensões das vias aéreas. Por menor que seja a dimensão da malha adotada, esta ainda não se torna representativa das vias aéreas, 
pois alguns trechos das paredes destas permanecem no interior dos volumes de controle, dificultando sua segmentação.

O MCA balão, em relação aos demais algoritmos, obtém o melhor desempenho considerando a soma das segmentações das categorias A e B, conforme ilustrado no gráfico da Figura 6, com o total de 83,33\% para estas categorias. O desempenho do MCA balão é seguido pelo CR, com $71,42 \%$, e pela rede MLP com $64,26 \%$ de segmentações para as mesmas categorias.

Apesar de apresentar as maiores taxas de segmentação da categoria A, a rede MLP e o CR não apresentam resultados satisfatórios paras as categorias D e E. No gráfico da Figura 7 é ilustrado o desempenho dos algoritmos, considerando-se as categorias D e E. Para estes resultados, o CR assim como o MCA balão apresentam poucas segmentações da categoria D. Porém, ao se considerar a categoria E, o MCA balão revela-se o melhor algoritmo, enquanto o CR ultrapassa a soma de segmentações das categorias $\mathrm{D}$ e E da rede MLP e do MCA topológico adaptativo.

Avaliando os resultados de acordo com as abordagens descritas, conclui-se que o MCA balão torna-se o algoritmo com melhor desempenho, por apresentar o maior percentual para o conjunto das

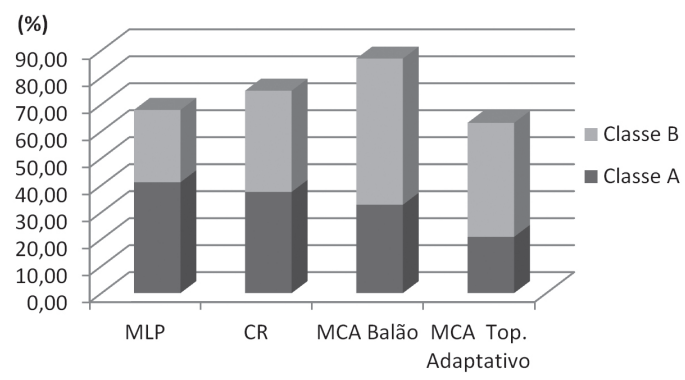

Figura 6. Gráfico de percentual das categorias A e B dos resultados da segmentação dos algoritmos avaliados.

Figure 6. Graphic for the percentage of classes $A$ and $B$ of the evaluated segmentation algorithms results.

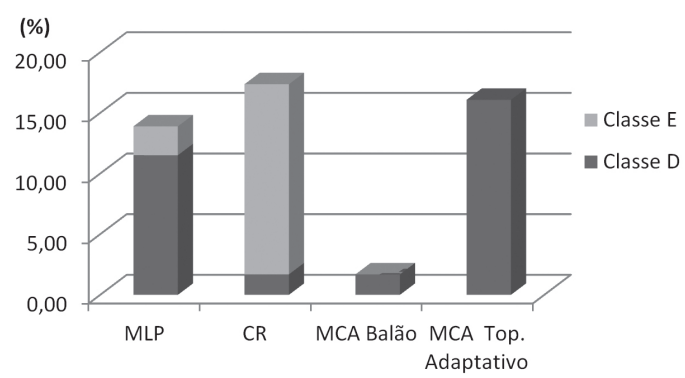

Figura 7. Gráfico de percentual das categorias D e E dos resultados da segmentação dos algoritmos avaliados.

Figure 7. Graphic for the percentage of classes $D$ and $E$ of the evaluated segmentation algorithms results. categorias A e B, a menor parcela de segmentação do tipo $\mathrm{D}$ e nenhuma segmentação do tipo $\mathrm{E}$. O algoritmo $\mathrm{CR}$, que apresenta uma parcela de segmentação da categoria A maior do que a do MCA balão, é o que possui maior parcela de segmentações da categoria $\mathrm{E}$.

A rede MLP apresentou a maior parcela de segmentações do tipo A, porém com parcelas consideráveis de segmentações das categorias D e E. O MCA topológico adaptativo revela-se um método mediano com algumas segmentações da categoria A e nenhuma da categoria $\mathrm{E}$.

\section{Avaliação do método de segmentação automática de vias aéreas}

Observando a Figura 5, percebe-se uma melhoria significativa em relação aos coeficientes de similaridade obtidos unicamente pelos algoritmos de detecção de mais de $10 \%$ para MLP e mais de $6 \%$ para ADP. Isto ocorre devido à redução significativa da quantidade de detecções FP.

Os métodos ADP/CR e MLP/CR são os que proporcionam as maiores reduções de detecções $\mathrm{FP}$, diminuindo de 83 para 7 e de 145 para 11 as detecções FP, respectivamente. Todavia, além de reduzir a quantidade de detecções $\mathrm{FP}$, o método $\mathrm{ADP} / \mathrm{CR}$, assim como o método MLP/CR, reduz significativamente a quantidade de acerto, devido ao vazamento presente na segmentação de algumas vias aéreas. Essa limitação é responsável pelo descarte de 77 detecções corretas do método de detecção baseado em ADP/CR e de 91 do método baseado em MLP/ $\mathrm{CR}$, o que impacta na taxa de sensibilidade como pode ser observado na Figura 5.

Os métodos com MCA não apresentam essa limitação, assim, nenhuma detecção correta foi descartada empregando-se estes métodos. Desta forma, mesmo apresentando uma redução menor de detecções FP, 65 e 117 detecções FP descartadas nos métodos ADP/MCA e MLP/MCA, respectivamente, as combinações dos algoritmos de detecção com o MCA são as que apresentam os maiores coeficientes de similaridade.

A baixa incidência de detecções FN nos resultados do algoritmo MLP, juntamente com a redução significativa de detecções FP e a manutenção das detecções corretas, proporcionadas pelo algoritmo MCA, resultam em coeficiente de similaridade de $91,3 \%$ e sensibilidade de $87,7 \%$.

Conforme descrito, este trabalho propõe métodos de segmentação automática das vias aéreas e os avalia, utilizando-se dos algoritmos MLP e ADP, para detectar as vias aéreas pulmonares em imagens $2 \mathrm{D}$ de TC do tórax, combinado com os algoritmos $\mathrm{CR}$, MCA balão e MCA topológico adaptativo para segmentá-las. 
Para avaliar estes métodos, é utilizado um conjunto de imagens composto por imagens de TCAR e TC multidetectores de voluntários sadios e pacientes com DPOC. Observa-se que, para o conjunto de imagens testadas, a detecção utilizando ADP e MLP, apresenta desempenho regular com CS de $80,6 \%$ e $81,2 \%$ e sensibilidade de $78,65 \%$ e $87,74 \%$, respectivamente. Deste modo, pode-se considerar que a MLP obteve melhor desempenho por apresentar coeficiente de similaridade e sensibilidade maiores. A avaliação de ambos os algoritmos deu-se pela comparação dos resultados com um padrão-ouro, obtidos por três médicos especialistas, sendo um pneumologista e dois radiologistas.

Em relação à segmentação, o algoritmo $\mathrm{CR}$ assim como o algoritmo MCA balão apresentaram poucas segmentações da categoria D. Porém, ao se considerar as segmentações da categoria E, o MCA balão revela-se o melhor. Ao considerar as segmentações das categorias A e B, o MCA balão também apresenta melhor desempenho.

Com os métodos de segmentação automática das vias aéreas, o coeficiente de similaridade tem melhorias significativas. Sendo possível detectar e eliminar boa parte dos FP. A baixa incidência de FNs, a redução de FPs e a manutenção das detecções corretas fazem do método MLP/MCA o de melhor desempenho obtendo CS de $91,3 \%$ e S de $87,7 \%$.

Desta forma, pode-se concluir que, para o conjunto de imagens utilizadas nos experimentos, o algoritmo MCA balão combinado com o algoritmo MLP, revela-se em um método que pode ser empregado para a segmentação automática das vias aéreas em imagens 2D de TC do tórax, podendo ser integrado a sistemas que auxiliem o diagnóstico médico, visto que esta combinação de algoritmos segmenta de forma automática e satisfatória as vias aéreas, permitindo posteriormente a extração de atributos que possibilitará a redução do tempo nas análises médicas.

\section{Agradecimentos}

Ao CNPq - Conselho Nacional de Pesquisa pelo apoio financeiro, aos Serviços de Pneumologia e de Imagem do Hospital Universitário Walter Cantídio da Universidade Federal do Ceará e aos pneumologistas e radiologistas que contribuíram diretamente com este trabalho.

\section{Referências}

Alexandria AR, Cortez PC, Felix JHS, Abreu JS. Método de contornos ativos pSnakes aplicado em imagens de ecocardiograma de eixo curto para cálculo de fração de ejeção. Revista Brasileira de Engenharia
Biomédica. 2011; 27(3):147-62. http://dx.doi.org/10.4322/ rbeb.2011.012

Bartz D, Mayer D, Fischer J, Ley S, Rio A, Thust S, Heussel CP, Kauczor HU, Straber W. Hybrid segmentation and exploration of the human lungs. In: VIS 2003: Proceedings of the 14th IEEE Visualization; 2003 Oct 24; Seattle. Washington: IEEE Computer Society; 2003. p. 177-84. http://dx.doi.org/10.1109/VISUAL.2003.1250370

Bauer C, Bischof H, Beichel R. Segmentation of airways based on gradient vector flow. In: IJCAI 2009: Proceedings of the Workshop on Explanation Aware Computing; 2009. IJCAI; 2009. p. 191-201.

Born S, Iwamaru D, Pfeifle M, Bartz D. 3-step segmentation of the lower airways with advanced leakage-control. In: IJCAI 2009: Proceedings of the Workshop on Explanation Aware Computing; 2009. IJCAI; 2009. p. 239-50.

Busayarat S, Zrimec T. Automatic detection of pulmonary arteries and assessment of bronchial dilatation in HRCT images of the lungs. In: ICSC 2005: Proceedings of the Congress on Computational Intelligence Methods and Applications; 2005; Istanbul. IEEE; 2005. p. 1-5. http:// dx.doi.org/10.1109/CIMA.2005.1662325

Campos HS, Lemos ACM. A asma e a DPOC na visão do pneumologista. Jornal Brasileiro de Pneumologia. 2009; 35(4). http://dx.doi.org/10.1590/S1806-37132009000400003

Cavalcante TS, Cortez PC, Rebouças Filho PP, Felix JHS, Holanda MA, Maia Filho JH, Costa NSS. ADP e MLP como métodos de detecção automática de vias aéreas em imagens $2 \mathrm{D}$ de tomografia computadorizada do tórax. In: CLAIB2011: Anais do V Congresso Latino-americano de Engenheira Biomédica; 2011 maio; Havana. Havana: Sociedad Cubana de Bioingeniería; 2011.

Cheng I, Nilufar S, Flores-Mir C, Basu A. Airway segmentation and measurement in $\mathrm{CT}$ images. Conference proceedings: Annual International Conference of the IEEE Engineering in Medicine and Biology Society. 2007; 2007:795-9.

Cohen LD. On active contour models and balloons. CVGIV: Image Understanding. 1991; 53:211-8. http://dx.doi.org/1 0.1016/1049-9660(91)90028-N

Dagher I, Tom KE. WaterBalloons: A hybrid watershed balloon snake segmentation. Image and Vision Computing. 2008; 26(7):905-12. http://dx.doi.org/10.1016/j. imavis.2007.10.010

Fabijanska A. Two-pass region growing algorithm for segmenting airway tree from MDCT chest scans. Computerized Medical Imaging and Graphics. 2009; 33(7):537-46. PMid:19473814. http:// dx.doi.org/10.1016/j.compmedimag.2009.04.012

Felix JHS, Cortez PC, Holanda MA, Colaço DF, Albuquerque VHC, Alexandria AR. Lung and chest wall structures segmentation in CT images. In: VIPIMAGE 2007: Proceedings of the 1st ECCOMAS Thematic Conference on Computational Vision and Medical Image Processing; 2007; London. London: Taylor and Francis/Balkema; 2007. p. 291-94.

Felix JHS, Cortez PC, Costa RCS, Fortaleza SCB, Pereira EDB, Holanda MA. Avaliação computacional de enfisema 
pulmonar em TC: comparação entre um sistema desenvolvido localmente e um sistema de uso livre. Jornal Brasileiro de Pneumologia. 2009; 35(9):868-76. PMid:19820813. http:// dx.doi.org/10.1590/S1806-37132009000900008

Felix JHS, Cortez PC, Alexandria AR, Holanda MA. The assessment of chronic obstructive pulmonary disease progression by computer tomography imaging analysis. In: IWSSIP 2010: Proceedings of the 17th International Conference on Systems, Signals and Image Processing; 2010; Rio de Janeiro. Rio de Janeiro: EdUFF; 2010. p. 121-24.

Gonzalez RC, Woods R. Digital Image Processing. 3rd ed. New Jersey: Prentice Hall, 2008.

Haykin S. Neural Networks: A comprehensive foundation. 2nd. ed. Ontario: Prentice-Hall, 1998. PMid:9697135.

Holanda MA, Fortaleza SCB, Almeida MA, Winkeler GFP, Reis RC, Felix JHS, Lima JWO, Pereira EDB. Continuous Positive Airway Pressure effects on regional lung aeration in patients with COPD: a high-resolution CT scan study. Chest. 2010; 138(2):305-14. PMid:20363847. http://dx.doi. org/10.1378/chest.09-2850

Irion KL, Hochhegger B, Marchiori E, Porto NS, Baldisserotto SV, Santana PR. Radiograma de tórax e tomografia computadorizada na avaliação do enfisema pulmonar. Jornal Brasileiro de Pneumologia. 2007; 33(6):720-32. PMid:18200374. http://dx.doi.org/10.1590/ S1806-37132007000600017

Irving B, Taylor P, Todd-Pokropek A. 3D segmentation of the airway tree using a morphology based method. In: IJCAI 2009: Proceedings of the Workshop on Explanation Aware Computing; 2009. IJCAI; 2009. p. 297-307.

Jannin P, Krupinski E, Warfield S. Validation in medical image processing. IEEE Transactions On Medical Imaging. 2006; 25(11):1405-9. PMid:17117769. http:// dx.doi.org/10.1109/TMI.2006.883282

Kaftan JN, Kiraly AP, Bakai A, Das M, Novak CL, Aach T. Fuzzy pulmonary vessel segmentation in contrast enhanced CT data. SPIE Proceedings. 2008; 6941Q. http://dx.doi. org/10.1117/12.768795

Kass M, Witkin A, Terzopoulos D. Snakes: active contours models. International Journal of Computer Vision. 1988; 1(4):321-31. http://dx.doi.org/10.1007/ BF00133570

Kiraly AP, Higgins WE, Mclennan G, Hoffman EA, Reinhardt JM. Three-dimensional human airway segmentation methods for clinical virtual bronchoscopy. Academic Radiology. 2002; 9(10):1153-68. http://dx.doi.org/10.1016/ S1076-6332(03)80517-2

Lee M, Park SY, Cho W, Kim S. Medical image segmentation using a geometric active contour model based on level set method. Journal of Korean Medical Science. 2007 June; 22(3):577-9. PMCid:PMC2693660.

Lo P, Bruijne M. Voxel classification based airway tree segmentation. IEEE Medical Imaging. 2008; 6914:69141K-12.

Lo P, Sporring J, Ashraf H, Pedersen JJH, Bruijne M. Vessel-guided airway tree segmentation: A voxel classification approach. Medical Image Analysis. 2010; 14:527-38. PMid:20395163. http://dx.doi.org/10.1016/j. media.2010.03.004

Madeiro JPV, Cortez PC, Marques JAL. Análise comparativa de desempenho das transformadas Wavelet e Hilbert na detecção do QRS em ECG. Revista Brasileira de Engenharia Biomédica. 2009; 25(3):153-66. http://dx.doi.org/10.4322/ rbeb.2012.072

McInerney T, Terzopoulos D. T-snakes: Topology adaptive snakes. Medical Image Analysis. 1999. 4(2):73-91. http:// dx.doi.org/10.1016/S1361-8415(00)00008-6

Mendoza CS, Acha B, Serrano C. Maximal contrast adaptive region growing for $\mathrm{CT}$ airway tree segmentation. In: IJCAI 2009: Proceedings of the Workshop on Explanation Aware Computing; 2009. IJCAI; 2009. p. 285-95.

Mori K, Hasegawa J, Suenaga Y, Toriwaki J. Automated anatomical labeling of the bronchial branch and its application to the virtual bronchoscopy system. IEEE Transactions on Medical Imaging. 2000; 19(2):103-14. PMid:10784282. http://dx.doi.org/10.1109/42.836370

Murata N, Yoshizawa S, Amari SI. Determining the number of hidden units for an artificial neural network model. IEEE Transactions on Neural Networks. 1994; 5(6):865-72. PMid:18267861. http://dx.doi.org/10.1109/72.329683

Nakano Y, Muro S, Sakai H, Hirai T, Chin K, Tsukino M, Nishimura K, Itoh H, Paré PD, Hogg JC, Mishima M. Computed tomographic measurements of airway dimensions and emphysema in smokers. American Journal of Respiratory and Critical Care Medicine. 2000; 162:1102-8. PMid:10988137. http://dx.doi.org/10.1164/ ajrccm.162.3.9907120

Nixon MS, Aguado AS. Feature Extraction and Image Processing for Computer Vision. 3rd. ed. Oxford: Elsevier; 2012.

Nohama P, Jorge RF, Valenga MH. Efeitos da estimulação diafragmática transcutânea sincronizada em pacientes com doença pulmonar obstrutiva crônica (DPOC): um estudo piloto. Revista Brasileira de Engenharia Biomédica. 2012; 28(2):103-15.

Organização Mundial de Saúde - OMS. Global strategy for the diagnosis, management and prevention of chronic obstrutive pulmonary disease. OMS; 2008.

Rebouças Filho PP, Cortez PC, Holanda MA. Modelo de Contorno Ativo Crisp: nova técnica de segmentação dos pulmões em imagens de TC. Revista Brasileira de Engenharia Biomédica. 2011; 27(4):259-72. http://dx.doi. org/10.4322/rbeb.2011.021

Rizi FY, Ahmadian A, Sahba N, Tavakoli V, Alirezaie J, Fatemizadeh E, Rezaie N. A hybrid fuzzy based algorithm for 3d human airway segmentation. In: ICBBE 2008: Proceedings of the Bioinformatics and Biomedical Engineering; 2008; Shanghai. IEEE; 2008a. p. 2295-8. http://dx.doi.org/10.1109/ICBBE.2008.906

Rizi FY, Ahmadian A, Fatemizadeh E, Alirezaie J. An optimization based approach embedded in a fuzzy connectivity algorithm for airway tree segmentation. Conference proceedings: Annual International Conference 
of the IEEE Engineering in Medicine and Biology Society. 2008; 2008:4011-4. http://dx.doi.org/10.1109/ IEMBS.2008.4650089

Sardanelli F, Giuseppetti GM, Panizza P, Bazzocchi M, Fausto A, Simonetti G, Lattanzio V, Maschio AD. Sensitivity of mri versus mammography for detecting foci of multifocal, multicentric breast cancer in fatty and dense breasts using the whole-breast pathologic examination as a gold standard. American Journal of Roentgenology. 2004; 183:1149-57. PMid:15385322. http://dx.doi.org/10.2214/ajr.183.4.1831149

Shi H, Scarfe WC, Farman AG. Upper airway segmentation and dimensions estimation from cone-beam $\mathrm{CT}$ image datasets. International Journal of Computer Assisted Radiology and Surgery. 2006; 1(3):177-86. http://dx.doi. org/10.1007/s11548-006-0050-8

Silva JSS. Segmentação Pulmonar em Estudos de Tomografia Axial Computorizada [dissertação]. Aveiro: Universidade de Aveiro; 2005. 182 p.

Tschirren J, Yavarna T, Reinhardt JM. Airway segmentation framework for clinical environments. In: IJCAI 2009: Proceedings of the Workshop on Explanation Aware Computing; 2009. IJCAI; 2009. p. 227-38.

Vieira SRR, Puybasset L, Richecoeur J, Lu Q, Cluzel P, Gusman PB, Coriat P, Rouby J.J. A lung computed tomographic assessment of positive end-expiratory pressure-induced lung overdistension. American Journal of Respiratory and Critical Care Medicine. 1998;158:1571-7. PMid:9817710. http:// dx.doi.org/10.1164/ajrccm.158.5.9802101
Webb WC, Brant WE, Major NM. Fundamentals of Body CT. 3rd ed. Philadelphia: Saunders, Elsevier Health Science; 2006.

Weinheimer O, Achenbach T, Düber C. Fully automated extraction of airways from CT scans based on self-adapting region growing. In: IJCAI 2009: Proceedings of the Workshop on Explanation Aware Computing; 2009. IJCAI; 2009. p. $315-21$.

Xu C, Prince JL. Snakes, shapes, and gradient vector flow. IEEE Transactions on Medical Processing. 1998;7(3):359-69. PMid:18276256. http://dx.doi.org/10.1109/83.661186

Zaidi H, Naqa IE. Pet-guided delineation of radiation therapy treatment volumes: a survey of image segmentation techniques. European Journal of Nuclear Medicine and Molecular Imaging. 2010;37(11):2165-87. PMid:20336455. http://dx.doi.org/10.1007/s00259-010-1423-3

Zijdenbos AP, Dawant BM, Margolin RA, Palmer AC. Morphometric analysis of white matter lesions in $\mathrm{mr}$ images: Method and validation. IEEE Transactions on Medical Imaging. 1994;13(4). PMid:18218550. http:// dx.doi.org/10.1109/42.363096

Zou KH, Warfield SK, Bharatha A, Tempany CMC, Kaus MR, Haker SJ, Wells WM, Jolesz FA, Kikinis R. Statistical Validation of Image Segmentation Quality Based on a Spatial Overlap Index. Academic Radiology. 2004;11(2):178-89. http://dx.doi.org/10.1016/S1076-6332(03)00671-8

\footnotetext{
Autores

Tarique da Silveira Cavalcante*, Paulo César Cortez, Thomaz Maia de Almeida

Departamento de Engenharia de Teleinformática, Universidade Federal do Ceará - UFC, Campus do PICI, B1. 725, CEP 60455-970, Fortaleza, CE, Brasil.

John Hebert da Silva Felix

Departamento de Energias, Universidade da Integração Internacional da Lusofonia Afro-Brasileira - UNILAB, Redenção, CE, Brasil.
} 